Nig. J. Biotech. Vol. 37(2): 177-193 (Dec. 2020)

ISSN: 01891731

Available online at

http://www.ajol.info/index.php/njb/index

and www.biotechsocietynigeria.org

DOI: https://dx.doi.org/10.4314/njb.v37i2.18

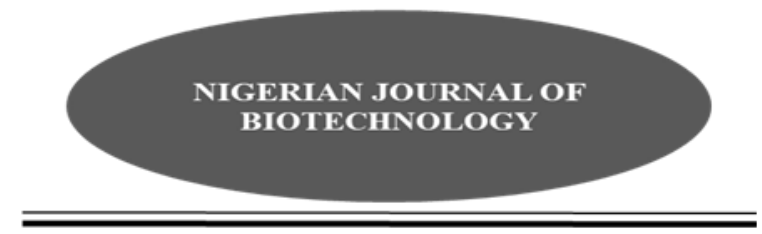

\title{
In-Vitro Antagonistic Effect of Bacillus thuringiensis on Ralstonia solanacearum, the Causal Agent of Bacterial Wilt Disease of Tomato (Lycopersicon esculentum Mill).
}

\author{
${ }^{* 2}$ C. O. OJESOLA, ${ }^{1}$ A. K. AKINTOKUN, ${ }^{3}$ P. O. AKINTOKUN AND ${ }^{1}$ A. R. OLOYEDE \\ ${ }^{1}$ Microbiology Department, Federal University of Agriculture, Abeokuta, Ogun State, Nigeria. \\ ${ }^{2}$ Biotechnology Centre, Federal University of Agriculture, Abeokuta, Ogun State, Nigeria. \\ ${ }^{3}$ Department of Plant Physiology and Crop Production, Federal University of Agriculture, Abeokuta, Ogun State, Nigeria.
}

\begin{abstract}
Tomato (Lycopersicon esculentum, Mill) is a rich source of vitamins, minerals and lycopene, which has many health benefits. However, its production is hampered by bacterial wilt caused by Ralstonia solanacearum resulting in significant yield losses. Use of chemicals in the control of plant pathogens has detrimental effects on humans and the environment in terms of leaving residues in soil which later find their way into underground waters. Therefore, it is desirable to find an alternative to chemical control of this bacterial pathogen. This study investigates the potential of native Bacillus thuringiensis (Bt) for biological control of Ralstonia solanacearum (Rs) under laboratory conditions. B. thuringiensis was isolated from cultivated soil, non- cultivated soil, stagnant water, sawdust, horse dung, grain dust, dead leaves and poultry manure. $R$. solanacearum was isolated from stem exudates of bacterial wilt infected plants and its pathogenicity assay was carried out using 2-week-old seedlings of Beske tomato variety. The Bt and $R$. solanacearum isolates were then characterized phenotypically. Bt isolates were further identified using endospore and parasporal staining techniques. All the Bt isolates were tested for in-vitro antagonistic activity on $R$. solanacearum using agar well diffusion method. Isolates Bt2, Bt16, Bt17, Bt32 and Bt34 were confirmed as Bacillus thuringiensis while isolate Rs was confirmed as $R$. solanacearum. Beske showed wilting symptoms from the fourth day of inoculation and eventual death of seedlings. The zone of inhibition exhibited ranged from $0.0 \mathrm{~mm}$ to $20.0 \mathrm{~mm}$.
\end{abstract}

Keywords: Bacillus thuringiensis, In-vitro, Bacterial wilt, Ralstonia solanacearum, Tomato.

*Corresponding author: idit01@yahoo.com Tel: +2348027320685 Introduction

Tomato (Lycopersicon esculentum Mill) belongs to the Solanaceae family (Khokar, 2013). It is widespread throughout the world and represents the most economically important vegetables even in Nigeria (Olaniyi et al., 2010; Bergougnoux,2013).Tomatoes contribute to a healthy, well-balanced diet. They are rich in minerals, vitamins, essential amino acids, sugars and dietary fibers. Tomato contains vitamin B and $C$, iron and phosphorus; its fruits are consumed fresh in salads or cooked in sauces, soup and meat or fish dishes.
They can be processed into purées, juices and ketchup. Yellow tomatoes have higher vitamin A content than red tomatoes, but red tomatoes contain lycopene, an antioxidant that may contribute to protection against carcinogenic substances (Khokar, 2013).

Recently, it has been estimated that huge proportions of vegetable crops get deteriorated annually during growth or post-harvest storage, owing to diseases caused by fungi, nematodes, 
bacteria, and viruses. This is one of the major limiting factors influencing food production and human development over thousands of years (Dun-chun et al., 2016).Bacterial wilt is a common bacterial disease in tropical, subtropical and some temperate regions of the world (Fegan and Prior, 2005). It is endemic in most tomato-growing areas of Nigeria, causing 60 to $100 \%$ yield losses (Popoola et al., 2015).

For the past 50 years, application of chemical pesticides has been the prevailing control measure for disease management in vegetables and other crops production. The continuous exposure to chemical pesticides adversely affects productivity, soil texture, nutritional content of vegetables, as well as the health of human beings (Singh et al., 2017). Due to the hazards associated with chemically synthesized herbicides and pesticides, management of diseases via biological control method is the novel emerging technology gaining importance in better agricultural sustainability (Singh et al ., 2017).

Farmers are shifting towards eco-friendly technologies for the management of pests and diseases, i.e., Biological Control Agents (BCAs) or BCA - based formulations, commonly called "Biopesticides". Examples include Trichoderma spp., Pseudomonas spp., Bacillus spp., Agrobacterium radiobacter, non- pathogenic Fusarium spp., Coniothyrium spp., Aspergillus niger, Bacillus thuringiensis (Bt), Metarthizium spp., Beauveria bassiana and nuclear polyhedrosis virus (NPVs), which are popularly used in plant protection (Keswani et al., 2015; Mishra et al., 2015).

Bacillus thuringiensis ( $B t)$, a spore-forming bacterium is well known for its insecticidal properties, associated with its ability to produce crystal inclusions during sporulation. These inclusions are proteins encoded by crygenes and have shown to be toxic to a variety of insects and other organisms like nematodes and protozoa (Koneckaet al., 2007). Formerly, only the insecticidal properties of $B$. thuringiensis attracted extensive attention. However, in recent years, the roles of $B$. thuringiensis in plant disease control have been discovered. Apart from crystal protein and other insecticidal substances, B. thuringiensis also produces other active components with good prospects for application as the following: zwittermicin A, which is extremely efficient at preventing the damping-off of alfalfa caused by Phytophthor amedicaginis (SiloSuhet al., 1994), and acyl homoserine lactone (AHL) lactonases, which can quench bacterial pathogenicity (Dong et al.,2002). Therefore, there is a need to assess the in-vitro antagonistic effect of Bacillus thuringiensis on Ralstonia solanacearum.

\section{Materials and methods}

\section{Study area and collection of soil samples}

The study covered 3 locations within the village extension mandate of the Federal University of Agriculture, Abeokuta (FUNAAB), Ogun State, Nigeria. These are FUNAAB main campus, Owe village and Obantoko. Samples from cultivated soil, non-cultivated soil, dead leaves, dead insects, stagnant water, grain dust, saw dust, poultry manure and horse dung were collected aseptically. Diseased tomato plants showing symptoms of bacterial wilt were also collected. The samples were collected into Ziploc bags, kept on ice packs and transported to the Microbiology Laboratory, FUNAAB.

\section{Isolation of Bacillus thuringiensis (Bt)}

\section{Isolation from soil}

Bacillus thuringiensis was isolated using the method described by Palma (2015) with slight modifications. Vegetative cells from sporulated bacteria were isolated by homogenizing $3 \mathrm{~g}$ of each soil sample in $10 \mathrm{ml}$ of sterile distilled water, intensely vortexed and mixed for 1 minute and incubated at $70 \pm 2^{\circ} \mathrm{C}$ for 15 minutes. After which the samples were vortexed and heated again. Each sample was then subjected to ten-fold dilutions and $20 \mu \mathrm{l}$ (from $10^{-3}$ to $10^{-5}$ ) was dispensed on nutrient agar. Plates were incubated at $28 \pm 2^{\circ} \mathrm{C}$ for at least $72 \mathrm{hrs}$ (Suguna et al., 2011).

\section{Isolation from stagnant water}

Ten milliliter $(10.0 \mathrm{ml})$ of each stagnant water sample was dispersed in $90 \mathrm{ml}$ of saline solution. This was left to equilibrate for 20 minutes and then subjected to heating in a water bath at $80 \pm 2^{\circ} \mathrm{C}$ for 10 minutes (Saadeldin, 2007). Each sample was then subjected to ten-fold dilutions and $20 \mu \mathrm{l}$ (from $10^{-3}$ to $10^{-5)}$ was dispensed on nutrient agar. Plates were incubated at $28 \pm 2^{\circ} \mathrm{C}$ for at least 72 hrs (Suguna et al., 2011).

\section{Isolation from insects, grain dust, animal dung and dead leaves}

Approximately $5 \mathrm{~g}$ of dead insects, saw dust, grain dust, animal dung and dead leaves were weighed 
separately into $95 \mathrm{ml}$ sterile distilled water in 250 $\mathrm{ml}$ conical flasks and incubated in an orbital shaker set at $25 \pm^{\circ} \mathrm{C}$ for $5 \mathrm{hr}$. One milliliter $(1 \mathrm{ml})$ aliquot was transferred into $5 \mathrm{ml}$ pre-warmed boiling tubes. The tubes were given heat-shock treatments at $80 \pm^{\circ} \mathrm{C}$ for 15 minutes, in a water bath. Each sample was then subjected to ten-fold dilutions and $20 \mu \mathrm{l}$ (from $10^{-3}$ to $10^{-5)}$ was dispensed on nutrient agar. Plates were incubated at $28 \pm 2^{\circ} \mathrm{C}$ for at least 72 hrs (Suguna et al., 2011).

\section{Isolation of Ralstonia solanacearum}

Ralstonia solanacearum was isolated as described by Shew and Lucas (1991) with slight modifications. A stem section was cut from diseased plants with vascular discoloration using a sterile sharp blade. The section was swabbed with $70 \%$ ethanol and subsequently placed against the inside wall of a water- filled clear test-tube so that the end of the section slightly touches the water surface. Milky white strands containing bacteria and extracellular polysaccharide streaming from the cut ends of the xylem were then cultured on nutrient agar plates incubated at $28 \pm 2^{\circ} \mathrm{C}$ for at least 24 hrs (Jayesh et al., 2014). Smooth, circular, raised and dirty- white colonies were sub-cultured to obtain pure cultures. The isolates were kept on nutrient agar slants maintained at $4^{\circ} \mathrm{C}$.

\section{Characterization of Bacterial isolates}

The bacterial isolates were subjected to standard microbiological methods such as morphological characteristics of the colony (shape, size, elevation, colour) and Gram staining to differentiate Gram negative and positive bacteria. Biochemical tests including catalase, oxidase, citrate utilization, VogesProskauer and methyl-red were carried out on the isolates (Fawole and Oso, 1998; Cheesbrough, 2006). The morphological and biochemical characteristics were examined according to the Bergey's Manual of Determinative Bacteriology (Holt et al., 1994).

\section{Pathogenicity test}

Pathogenicity assay of the Ralstonia solanacearum isolates was carried out under screen house conditions by inoculating susceptible Beske tomato seedlings. Bacterial isolates were grown on nutrient agar medium for two days at $28 \pm 2^{\circ} \mathrm{C}$. The cells were removed by centrifugation at 7,000 rpm for 10 minutes; pellets were re suspended in sterile distilled water and adjusted to a final density of $1 \times$ $10^{7} / \mathrm{ml}$ (Elsharkawy et al., 2015). Tomato seeds were nursed in a plastic tray and transplanted into disposable plastic cups 10 days after planting, while the inoculum was introduced into healthy seedlings 2 days after, by drenching the soil with $10 \mathrm{ml}$ each of the suspected pathogens (Hyakumachi et al., 2013). Seedlings inoculated with sterile water served as negative control. Wilt intensity was calculated after 21 days of inoculation using the method described by Abeer and Hend (2013). $\mathrm{I} \%=\left[\sum(\mathrm{nixv} 1 /(\mathrm{VxN})] \times 100\right.$, where $\mathrm{I}=$ wilt intensity, ni $=$ no of plants with respective disease rating, $\mathrm{v} 1=$ disease rating (following scale : $1=$ no symptoms, $2=$ 1 leaf wilted, $3=2$ or 3 leaves wilted, $4=$ four or more leaves wilted, $5=$ plant dead), $\mathrm{V}=$ the highest disease rating and $\mathrm{N}=$ the no of plants observed.

\section{In-vitro activity}

In-vitro antagonistic activity on the pathogen was determined using agar well diffusion method in which about $10 \mu$ l of pathogen suspension $\left(1 \times 10^{7}\right.$

$\mathrm{cfu} / \mathrm{ml}$ ) was evenly spread on nutrient agar plates. Subsequently, with the aid of a sterile cork borer (6mm in size), wells of $20 \mathrm{~mm}$ apart and diameter of about $6 \mathrm{~mm}$ were punched aseptically. Antagonist suspension $\left(1.8 \times 10^{8} \mathrm{cfu} / \mathrm{ml}\right.$, about $\left.100 \mu \mathrm{l}\right)$, was added into each well. Wells inoculated with sterile distilled water served as control (Elsharkawy et al., 2015; Mounyr et al.,2016). This was carried out as follows: antagonist and pathogen were inoculated at the same time, pathogen was inoculated first followed by antagonist applied 48 hrs after, antagonist inoculated first while pathogen was applied $48 \mathrm{hrs}$ after. The plates were then incubated for 24 hrs at $30 \pm 2^{\circ} \mathrm{C}$ after which zones of inhibition were measured (Cleidson et al, 2007, Mounyr et al.,2016, Marissa et al., 2016).

\section{Molecular characterization of the Bacterial isolates}

Genomic DNA of antagonistic Bt and Rs were extracted using Bacterial DNA isolation kit (Norgen BIOTEK, USA) followed by amplification of $16 \mathrm{~S}$ rRNA gene in $10 \mu \mathrm{l}$ reaction mix $(3.1 \mu \mathrm{l}$ of nuclease free $\mathrm{H}_{2} \mathrm{O}, 1.0 \mu \mathrm{l}$ of 10 x buffer, $1.0 \mu \mathrm{l}$ of $25 \mathrm{mM} \mathrm{MgCl}_{2}, 0.8$ $\mu \mathrm{l}$ of $2.5 \mathrm{mM}$ DNTPs, $0.5 \mu \mathrm{l}$ of $5 \mathrm{pMol}$ forward primer, $0.5 \mu$ of 5 pMol forward primer, $1.0 \mu$ l of DMSO, 2.0 $\mu \mathrm{l}$ of $10 \mathrm{ng} / \mu \mathrm{l}$ DNA and $0.1 \mu \mathrm{l}$ of $5 \mathrm{ng} / \mu \mathrm{l}$ of Taq polymerase) using Forward primer $16 \mathrm{SF}$ ( $5^{\prime}-$ 
GTGCCAGCAGCCGCGCTAA-3') and Reverse primer 16SR (5'AGACCCGGGAACGTATTCAC-3') (Taiwo et al., 2017).

The conditions for Polymerase Chain Reaction (PCR) in the thermal cycler (GeneAmp PCR System 9600) were as follows: $94^{\circ} \mathrm{C}$ for 5 minutes followed by 36 cycles of denaturation at $94^{\circ} \mathrm{C}$ for $30 \mathrm{sec}$, annealing at $56^{\circ} \mathrm{C}$ for $30 \mathrm{sec}$, extension at $72^{\circ} \mathrm{C}$ for $45 \mathrm{sec}$, and final extension at $72^{\circ} \mathrm{C}$ for 7 minutes. The amplified fragments were resolved by electrophoresis on a $1.5 \%$ agarose gel prepared in $0.5 \mathrm{X} \mathrm{TBE}$, stained with Gel red using a 50bp ladder. The gel ran for 50 minutes at $100 \mathrm{~V}$ and was visualized under UV transilluminator. The PCR product of the amplified $16 \mathrm{~S}$ rRNA amplified region was purified using ethanol precipitation method.

Morphological and Biochemical characteristics of bacterial isolates

All the bacterial isolates were rod-shaped and motile. Isolate T3 had dirty - white color while others were cream colored. Some were large while others

Table 1: Morphological characteristics of the isolates
The PCR product was used for another PCR reaction (sequencing reaction), using the big Dye Terminator method with 3130xl genetic analyzer from Applied Biosystems. Amplification and sequencing were done at International Institute for Tropical Agriculture (IITA) Biosciences Laboratory, Ibadan, Oyo State.

\section{Sequence editing and database matching}

The sequences were edited, assembled and aligned using BioEditsoftware (version 7.1.9). Gene sequences were compared at the Genbank database of NCBI (National Centre for Biotechnology Information) using BLASTn search tool to identify the isolates. Phylogenetic analysis was done using Molecular Evolutionary Genetics Analysis (MEGA) version 6 (Tamura et al., 2013).

\section{Results}

were small in size. Some of the bacteria had raised elevation while others had flat elevation (Table 1). The bacterial isolates were Gram positive except T3 (Table 2).

\begin{tabular}{lllllll}
\hline ID & Shape & Motility & Colour & Elevation & Size & Probable organism \\
\hline 1 & Rod & Motile & Cream & Flat & Small & Bacillus species \\
2 & Rod & Motile & Cream & Flat & Big & Bacillus species \\
3 & Rod & Motile & Cream & Flat & Small & Bacillus species \\
4 & Rod & Motile & Cream & Flat & Small & Bacillus species \\
5 & Rod & Motile & Cream & Flat & Small & Bacillus species \\
6 & Rod & Motile & Cream & Flat & Small & Bacillus species \\
7 & Rod & Motile & Cream & Flat & Small & Bacillus species \\
8 & Rod & Motile & Cream & Flat & Small & Bacillus species \\
9 & Rod & Motile & Cream & Flat & Small & Bacillus species \\
10 & Rod & Motile & Cream & Flat & Small & Bacillus species \\
11 & Rod & Motile & Cream & Flat & Small & Bacillus species \\
12 & Rod & Motile & Cream & Flat & Small & Bacillus species \\
\hline
\end{tabular}


Ojesola et al. /Nig. J. Biotech. Vol. 37 Num. 2: 177-193 (Dec 2020)

\begin{tabular}{|c|c|c|c|c|c|c|}
\hline 13 & Rod & Motile & Cream & Flat & Small & Bacillus species \\
\hline 14 & Rod & Motile & Cream & Flat & Small & Bacillus species \\
\hline 15 & Rod & Motile & Cream & Flat & Small & Bacillus species \\
\hline 16 & Rod & Motile & Cream & Flat & $\mathrm{Big}$ & Bacillus species \\
\hline 17 & Rod & Motile & Cream & Flat & $\mathrm{Big}$ & Bacillus species \\
\hline 18 & Rod & Motile & Cream & Flat & Small & Bacillus species \\
\hline 19 & Rod & Motile & Cream & Flat & Small & Bacillus species \\
\hline 20 & Rod & Motile & Cream & Flat & Small & Bacillus species \\
\hline 21 & Rod & Motile & Cream & Flat & Small & Bacillus species \\
\hline 22 & Rod & Motile & Cream & Flat & Small & Bacillus species \\
\hline 23 & Rod & Motile & Cream & Flat & Small & Bacillus species \\
\hline 24 & Rod & Motile & Cream & Flat & Small & Bacillus species \\
\hline 25 & Rod & Motile & Cream & Flat & Small & Bacillus species \\
\hline 26 & Rod & Motile & Cream & Flat & Small & Bacillus species \\
\hline 27 & Rod & Motile & Cream & Flat & Small & Bacillus species \\
\hline 28 & Rod & Motile & Cream & Flat & Small & Bacillus species \\
\hline 29 & Rod & Motile & Cream & Flat & Small & Bacillus species \\
\hline 30 & Rod & Motile & Cream & Flat & Small & Bacillus species \\
\hline 31 & Rod & Motile & Cream & Flat & Small & Bacillus species \\
\hline 32 & Rod & Motile & Cream & Flat & Big & Bacillus species \\
\hline 33 & Rod & Motile & Cream & Flat & Small & Bacillus species \\
\hline 34 & Rod & Motile & Cream & Flat & Big & Bacillus species \\
\hline 35 & Rod & Motile & Cream & Flat & Small & Bacillus species \\
\hline 36 & Rod & Motile & Cream & Flat & Small & Bacillus species \\
\hline 37 & Rod & Motile & Cream & Flat & Small & Bacillus species \\
\hline 38 & Rod & Motile & Cream & Flat & Small & Bacillus species \\
\hline 39 & Rod & Motile & Cream & Flat & Small & Bacillus species \\
\hline 40 & Rod & Motile & Cream & Flat & Small & Bacillus species \\
\hline 41 & Rod & Motile & Cream & Flat & Small & Bacillus species \\
\hline
\end{tabular}


Ojesola et al. /Nig. J. Biotech. Vol. 37 Num. 2: 177-193 (Dec 2020)

\begin{tabular}{lllllll}
\hline 42 & Rod & Motile & Cream & Flat & Small & Bacillus species \\
43 & Rod & Motile & Cream & Flat & Small & Bacillus species \\
44 & Rod & Motile & Cream & Flat & Small & Bacillus species \\
45 & Rod & Motile & Cream & Flat & Small & Bacillus species \\
46 & Rod & Motile & Cream & Flat & Small & Bacillus species \\
47 & Rod & Motile & Cream & Flat & Small & Bacillus species \\
48 & Rod & Motile & Cream & Flat & Small & Bacillus species \\
49 & Rod & Motile & Cream & Flat & Small & Bacillus species \\
50 & Rod & Motile & Cream & Flat & Small & Bacillus species \\
51 & Rod & Motile & dirty- white & Raised & Small & Ralstonia solanacearum \\
52 & Rod & Motile & dirty- white & Raised & Small & Ralstonia solanacearum \\
\hline
\end{tabular}


Table 2: Biochemical characteristics of the bacterial isolates

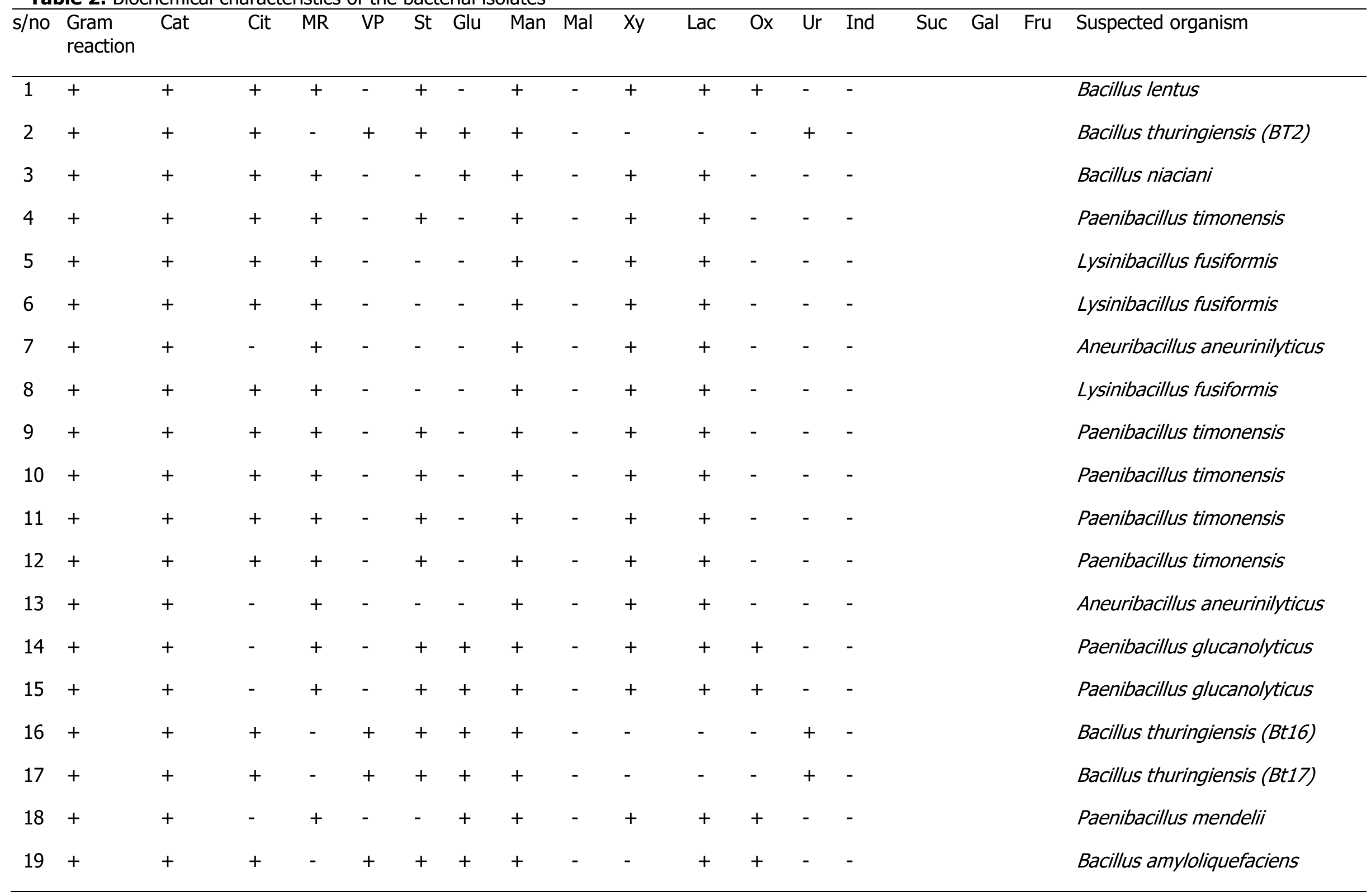


Ojesola et al. /Nig. J. Biotech. Vol. 37 Num. 2: 177-193 (Dec 2020)

\begin{tabular}{|c|c|c|c|c|c|c|c|c|c|c|c|c|c|c|c|}
\hline 20 & + & + & - & + & - & - & - & + & - & + & + & - & - & - & Aneuribacillus aneurinilyticus \\
\hline 21 & + & + & - & + & + & - & + & + & - & + & + & - & - & - & Bacillus pumilus \\
\hline 22 & + & + & - & + & + & - & + & + & - & + & + & - & - & - & Bacillus pumilus \\
\hline 23 & + & + & + & - & + & + & + & + & - & - & + & + & - & - & Bacillus amyloliquefaciens \\
\hline 24 & + & + & - & + & - & + & + & + & - & + & + & + & - & - & Paenibacillus glucanolyticus \\
\hline 25 & + & + & + & + & - & - & + & + & - & + & + & - & - & - & Bacillus niaciani \\
\hline 26 & + & + & - & + & - & - & - & + & - & + & + & - & - & - & Aneuribacillus aneurinilyticus \\
\hline 27 & + & + & + & + & - & - & + & + & - & + & + & - & - & - & Bacillus niaciani \\
\hline 28 & + & + & - & + & - & + & + & + & - & + & - & + & - & - & Bacillus smithii \\
\hline 29 & + & + & - & + & + & - & + & + & - & + & + & - & - & - & Bacillus pumilus \\
\hline 30 & + & + & - & + & - & + & - & + & - & + & + & + & - & - & Bacillus megaterium \\
\hline 31 & + & + & - & + & - & + & - & + & - & + & + & + & - & - & Bacillus megaterium \\
\hline 32 & + & + & + & - & + & + & + & + & - & - & - & - & + & - & Bacillus thuringiensis (Bt32) \\
\hline 33 & + & + & - & + & - & + & + & + & - & + & + & + & - & - & Paenibacillusglucanolyticus \\
\hline 34 & + & + & + & - & + & + & + & + & - & - & - & - & + & - & Bacillus thuringiensis (Bt34) \\
\hline 35 & + & + & - & + & + & + & + & + & - & - & - & + & - & - & Bacillus assamensis \\
\hline 36 & + & + & - & + & + & + & + & + & - & - & - & + & - & - & Bacillus muralis \\
\hline 37 & + & + & + & + & - & - & - & + & - & + & + & - & - & - & Lysinibacillus fusiformis \\
\hline 38 & + & + & + & + & - & + & + & + & - & - & + & - & - & - & Paenibacillus massilliensis \\
\hline 39 & + & + & + & + & - & + & - & + & - & + & + & - & - & - & Paenibacillus timonensis \\
\hline 40 & + & + & + & + & - & + & - & + & - & + & + & - & - & - & Paenibacillus timonensis \\
\hline 41 & + & + & - & + & + & - & + & + & - & + & + & - & - & - & Bacillus pumilus \\
\hline
\end{tabular}


Ojesola et al. /Nig. J. Biotech. Vol. 37 Num. 2: 177-193 (Dec 2020)

\begin{tabular}{|c|c|c|c|c|c|c|c|c|c|c|c|c|c|c|c|c|c|c|}
\hline 42 & + & + & - & + & + & - & + & + & - & + & + & - & - & - & & & & Bacillus pumilus \\
\hline 43 & + & + & - & + & - & + & + & + & - & + & - & + & - & - & & & & Bacillus smithii \\
\hline 44 & + & + & - & + & + & - & + & + & - & + & + & - & - & - & & & & Bacillus pumilus \\
\hline 45 & + & + & - & + & + & - & + & + & - & + & + & - & - & - & & & & Bacillus pumilus \\
\hline 46 & + & - & - & + & + & + & + & + & + & + & + & + & - & - & & & & Bacillus siamensis \\
\hline 47 & + & + & - & + & + & - & + & + & - & + & + & - & - & - & & & & Bacillus pumilus \\
\hline 48 & + & - & - & + & + & + & + & + & + & + & + & + & - & - & & & & Bacillus siamensis \\
\hline 49 & + & + & - & + & + & + & + & + & + & + & + & - & - & - & & & & Bacillus lichenfomis \\
\hline 50 & + & + & - & + & + & - & + & + & - & + & + & - & - & - & & & & Bacillus pumilus \\
\hline 51 & - & + & + & - & - & - & + & - & + & + & + & + & + & - & + & + & + & Ralstonia solanacearum (T1) \\
\hline 52 & - & + & + & + & + & - & + & - & + & + & + & + & + & - & + & + & + & Ralstonia solanacearum (T3) \\
\hline
\end{tabular}

Cat-catalase; Cit-citrate; MR-methyl-Red; VP-Voge-Proskauer; St-starch; Glu-glucose; Man-mannitol; Mal-maltose; Xy-xylose; Lac-lactose; Oxoxidase; Ur-urease; Ind- indole; Suc-sucrose; Gal-galactose; Fru-fructose.

+ Positive reaction; - Negative reaction 
Endospore and parasporal crystal staining for Bacillus thuringiensis identification
Isolates Bt2, Bt16, Bt17, Bt32, and Bt34 were positive for endospore and crystal staining (Table 3).

Table 3: Endospore and parasporal crystal staining of the bacterial isolates

\begin{tabular}{lcc}
\hline Bacterial isolate & Endospore staining & $\begin{array}{l}\text { Parasporal } \\
\text { staining }\end{array}$ \\
\hline Bt2 & & body \\
Bt16 & + & + \\
Bt17 & + & + \\
Bt32 & + & + \\
Bt34 & + & + \\
\hline
\end{tabular}

Percentage occurrence of Bacteria species isolated from different sources

Bacillus pumilus had the highest percentage occurrence $(53 \%)$ followed by Paenibacillus timonensis (41\%) while the least occurrence was observed in Paenibacillus mendelis, Bacillus assamensis, Bacillus muralis, Paenibacillus massilliensis and Bacillus licheniformis(6\%) as shown in Figure 1.

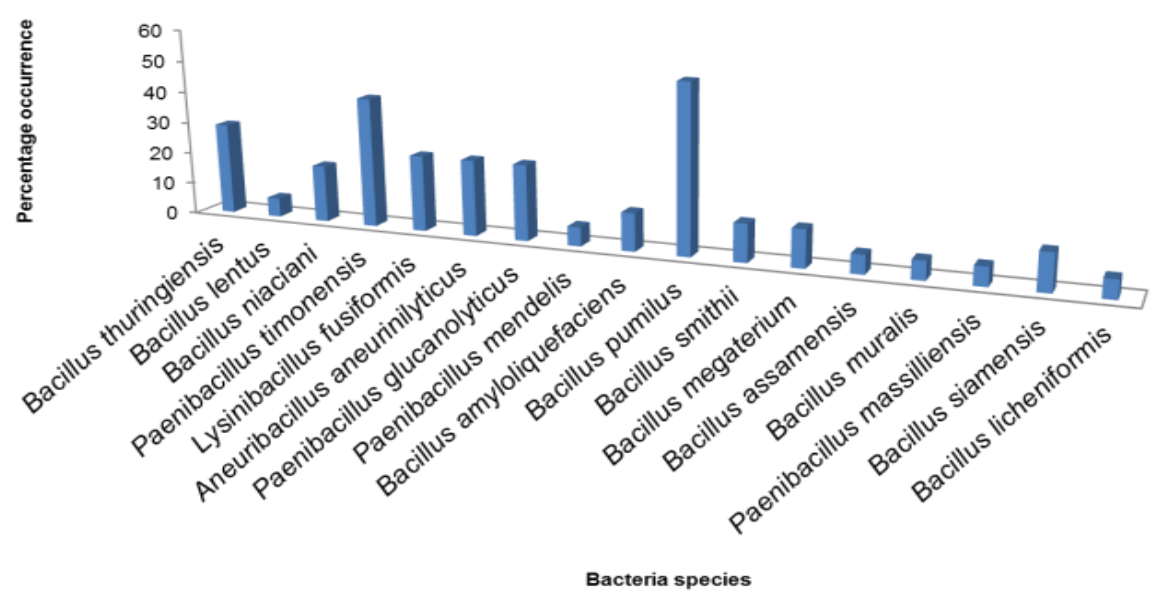

Fig. 1: Percentage occurrence of bacteria species isolated from different sources

Percentage occurrence of Bacillus thuringiensis isolated from different sources

Cultivated soil had the highest percentage occurrence of Bacillus thuringiensis $(60 \%)$ while the least occurrence was observed in stagnant water and dead insects (20\%) (Figure 2). 
Ojesola et al. /Nig. J. Biotech. Vol. 37 Num. 2: 177-193 (Dec 2020)

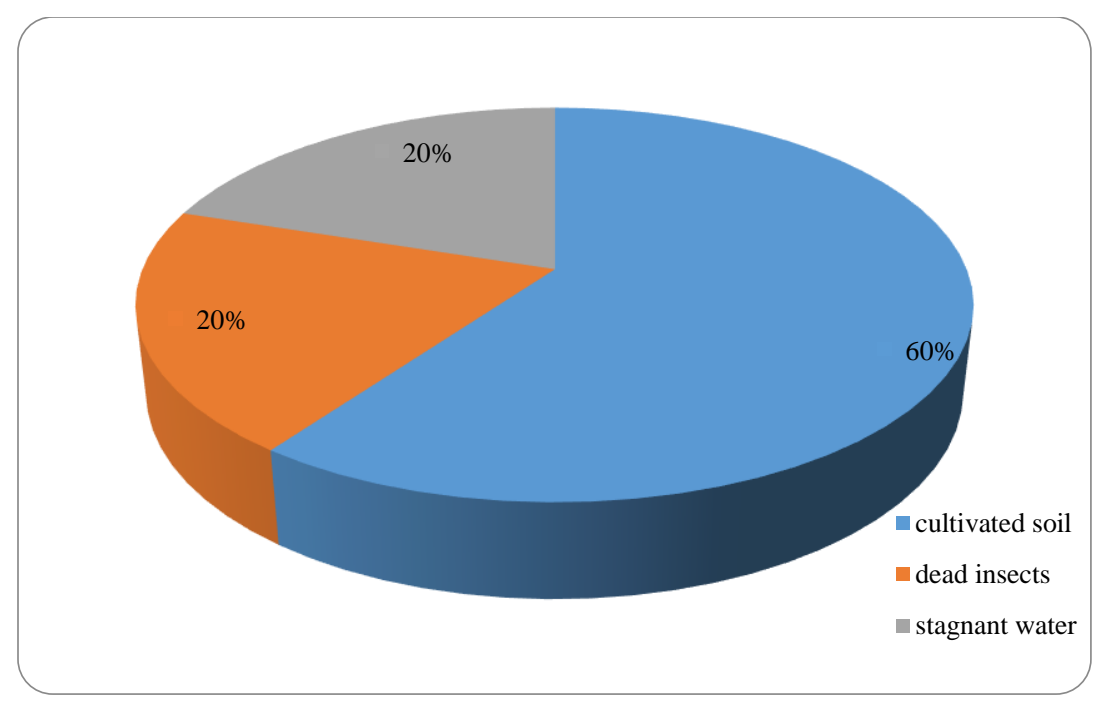

Figure 2: Percentage occurrence of Bacillus thuringiensis isolated from different sources

Pathogenicity

Wilting of varying degrees were observed on seedlings inoculated with pathogens while

In-vitro activity

There were no zones of inhibition when either antagonist or pathogen was applied first. When seedlings inoculated with sterile water showed no sign of wilting as shown in Table 3.

pathogen and antagonist were applied at the same time, isolate Bt34 had the highest zone of inhibition $(20 \mathrm{~mm})$ while isolate Bt17 had the least zone of inhibition (0 mm) (Table 4).

Table 3: Pathogenicity Test for Ralstonia solanacearum on healthy tomato seedlings

ID

\section{R1}

R2

R3

R4

R5

R6

C1

$\mathrm{C} 2$

C3

C4

\section{No of wilted leaves}

All (dead)

4

All (dead)

All (dead)

5

All (dead)

None

None

None

None 
C5

None

C6

None

KEY: R1-R6- seedlings with pathogen; C1-C6- seedlings with sterile water

Table 4: In-vitro antagonistic effect of Bacillus thuringiensis against Ralstonia solanacearum

\begin{tabular}{lc} 
ID & Zone of inhibition ( \\
\hline Bt2 & 4 \\
Bt16 & 2 \\
Bt17 & 0 \\
Bt32 & 1 \\
Bt34 & 20
\end{tabular}

Molecular Characterization of the Bacterial Isolates

Purity and concentration of DNA extracted from the bacterial isolates ranged from 1.74 to 1.92 and $32 \mathrm{ng} / \mu \mathrm{l}$ to $160.50 \mathrm{ng} \mu \mathrm{l}$, respectively, as shown in Table 5 . Similarity of sequences of the bacterial isolates with sequences obtained from NCBI is shown in Table 6.

Gel images of genomic DNA are shown in Plate 1 while Plate 2 shows the gel image of amplified $16 \mathrm{~S}$ rRNA gene of the isolates. Phylogenetic relationship of the bacterial isolates is shown in Figure 3.

Table 5: Purity and Concentration of the DNA extracted from the bacterial isolates

\begin{tabular}{lll}
\hline Sample & Purity & Concentration $(\mathbf{n g} / \boldsymbol{\mu l})$ \\
\hline RsT1 & 1.74 & 130.00 \\
RsT3 & 1.86 & 32.40 \\
Bt2 & 1.82 & 145.40 \\
Bt34 & 1.92 & 160.50 \\
\hline
\end{tabular}

KEY: RsT1 - Ralstonia solanacearum; RsT3 - Ralstonia solanacearum; Bt2 - Bacillus thuringiensis; Bt34 - Bacillus thuringiensis

Table 6: Similarity of the sequences from the Bacterial strains with sequences obtained from NCBI genbank database.
Bacterial isolate
Closest related taxa
$\%$ Similarity
Accession Number 
Ojesola et al. /Nig. J. Biotech. Vol. 37 Num. 2: 177-193 (Dec 2020)

\begin{tabular}{llll}
\hline Ralstonia solanacearum(T1) & Ralstonia solanacearum APK76 & $95 \%$ & MF973211.1 \\
Ralstonia solanacearum (T3) & Ralstonia solanacearum Rs8 & $95 \%$ & HRG425352.1 \\
Bacillus thuringiensis (Bt2) & $\begin{array}{l}\text { Bacillus thuringiensis strain INF- } \\
71\end{array}$ & $85 \%$ & KP813739.1 \\
Bacillus thuringiensis (Bt34) & $\begin{array}{l}\text { Bacillus thuringiensis strain PKN } \\
\text { B }\end{array}$ & $81 \%$ & KF922484.1
\end{tabular}

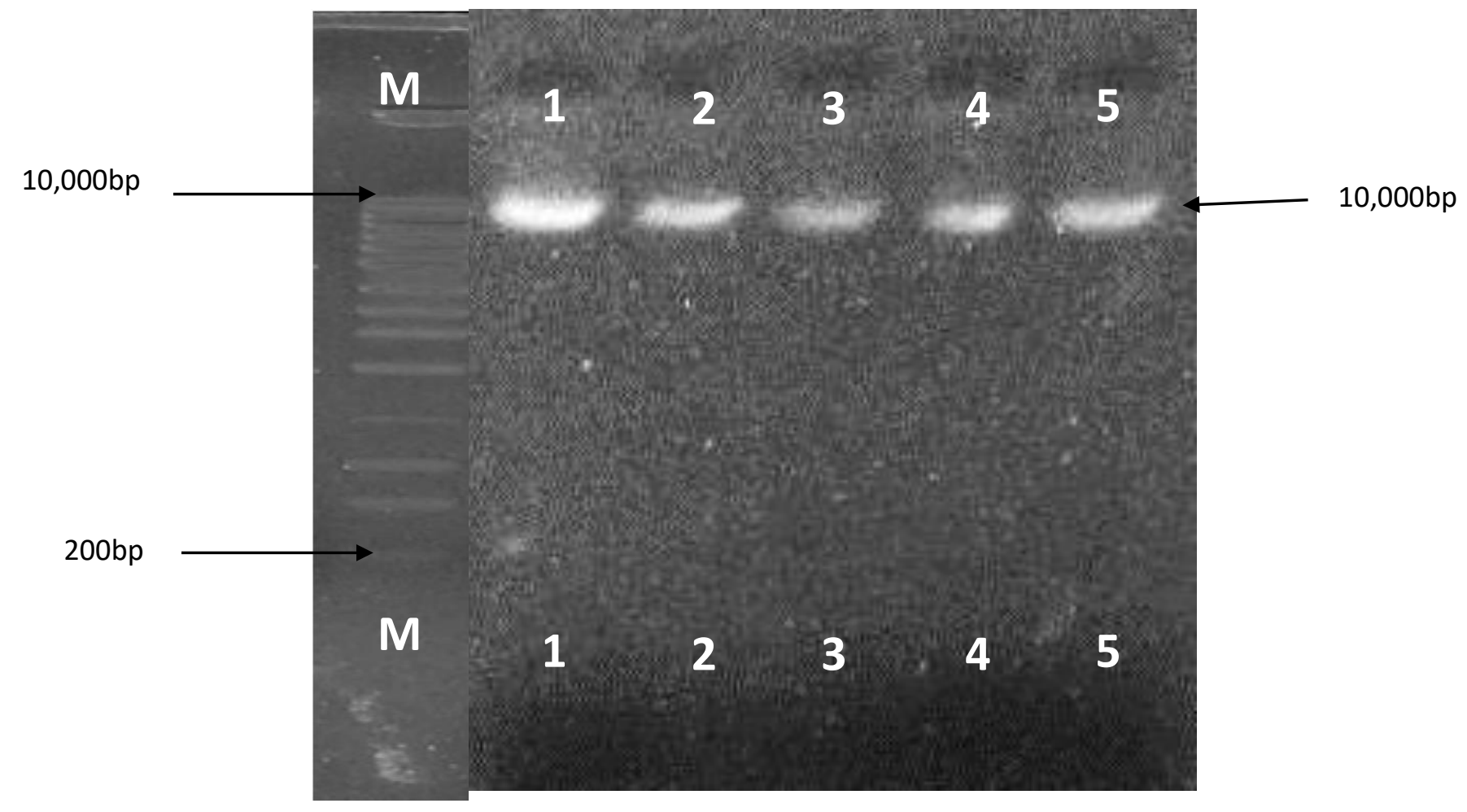

Plate 1: Agarose gel electrophoresis of genomic DNA

M- Molecular ladder; 1-Ralstonia solanacearum (T1); 2-Ralstonia solanacearum (T3) 4-Bacillus thuringiensis 


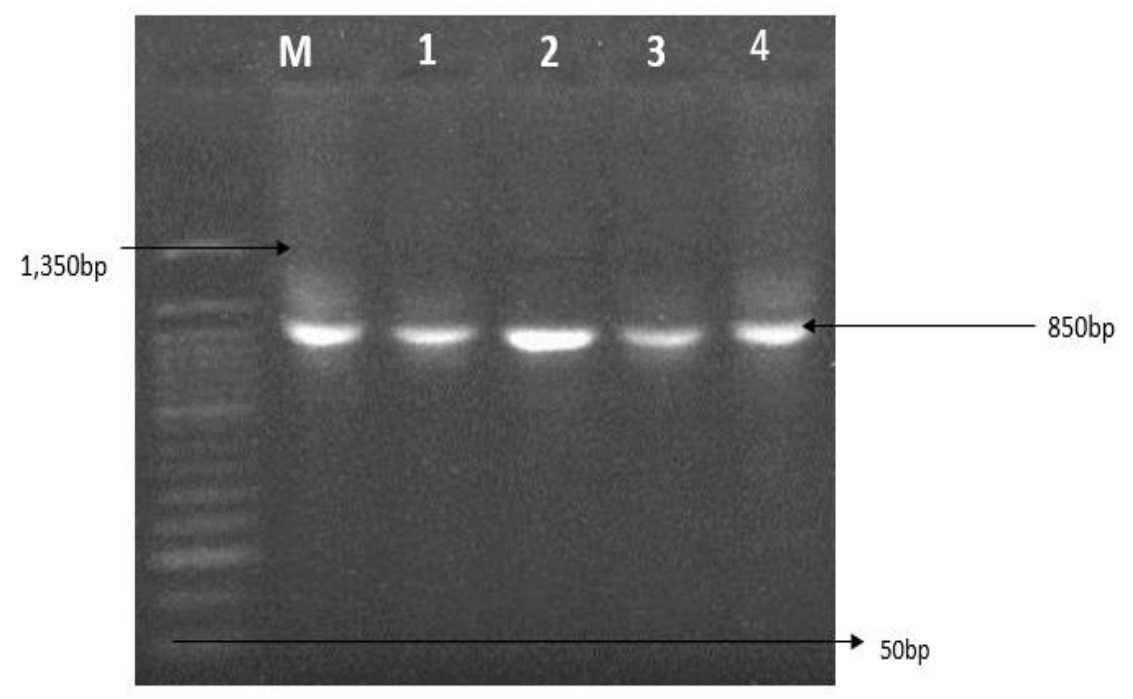

Plate 2: Agarose gel electrophoresis showing amplified 16S rRNA gene of the isolates M- Molecular ladder; 1: Ralstonia solanacearum(T1); 2: Ralstonia solanacearum(T3) 4- Bacillus thuringiensis (Bt2); 5- Bacillus thuringiensis (Bt34)

Figure 3: Phylogenetic relationship of the bacterial isolates

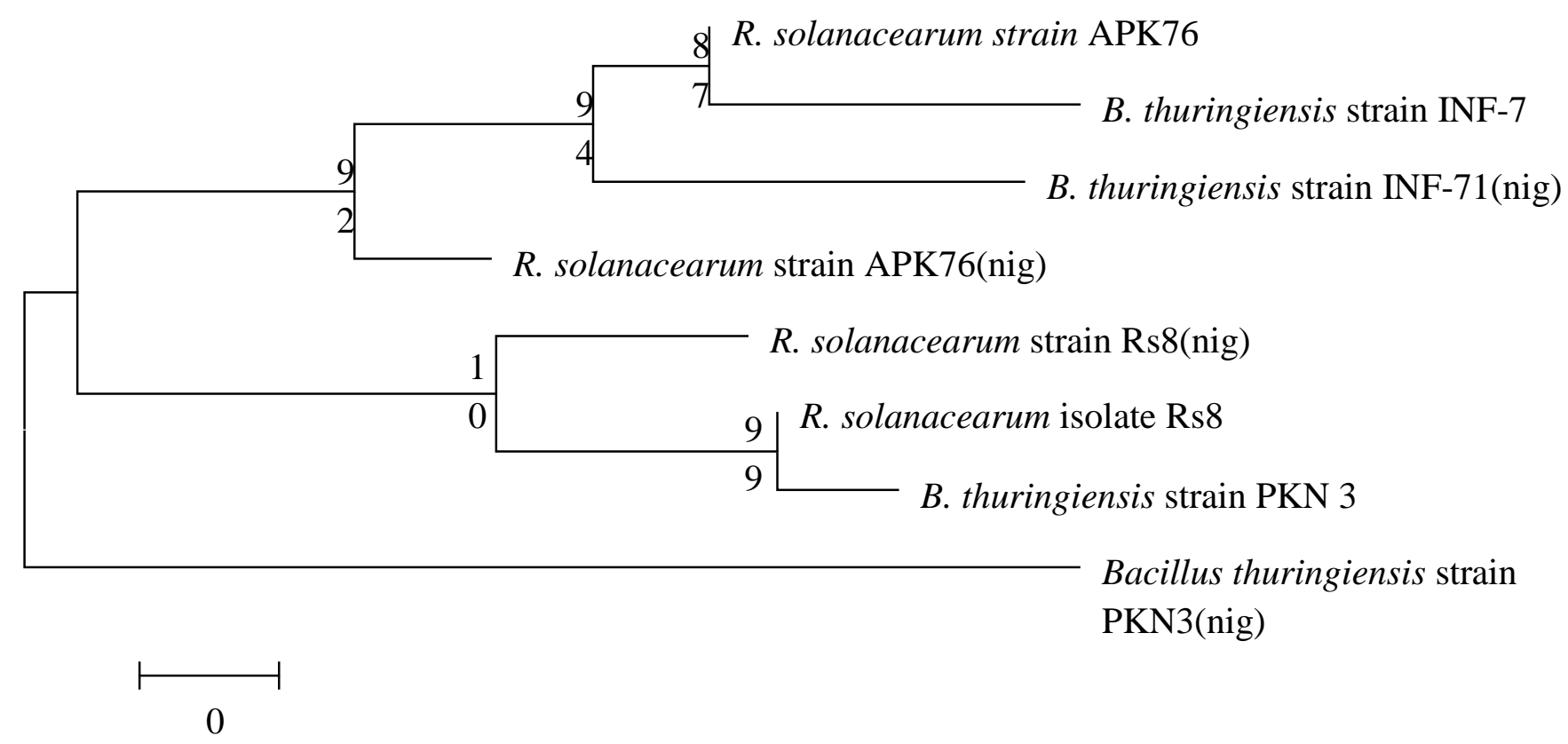




\section{Discussion}

Biocontrol is particularly desirable because it is sustainable, environment friendly, cost-effective and could be used in integrated pest management programs (Elshakawy et al, 2015). In this study, 17 bacterial isolates were isolated from cultivated and non-cultivated soils, stagnant waters, dead leaves and dead insects. This corroborates the findings of Argôlo-Filho and Loguercio, (2013), El-kersh et al.,(2016), and Denane et al., (2017), that were able to isolate Bt from different sources. One bacterial isolate was also isolated from diseased tomato plants showing symptoms of bacterial wilt. Based on their morphological and biochemical characteristics, 5 out of the 17 bacterial isolates (Bt2, Bt16, Bt17, Bt32 and Bt34) recovered from the different sources (cultivated soils, dead insects and stagnant waters) were tentatively identified as Bacillus thuringiensis (Bt). Endospore and crystal staining further confirmed the Bt isolates. This corroborates the works of Mohsina et al., (2013), Palma (2015) and Meihiaret al., (2015). They were able to establish Bt as crystal protein and endospore producers. Ammons et al.,(2016) and Neethu et al.,(2015) also reported that crystal proteins account for their pesticidal and insecticidal activities. Out of the 17 different Bacillus species isolated from the different sources, Bacillus pumilus occurred most, this could be as a result of their spores' extreme resistance to radiation, desiccation, and hydrogen peroxide treatment as reported by Link et al., (2004) and Kempf, et al., (2005). As a result, $B$. pumilus has been classified as an extreme microorganism according to the planetary protection standards (Vaishampayan et al., 2012). Bacillus thuringiensis had the highest percentage occurrence in cultivated (agricultural) soil samples; this contradicts the findings of Kassougue et al., (2015) but agrees with that of Ralte et al., (2016). Kassogue found low Bt strains in cultivated soil in Mali while Ralte found higher frequency of Bacillus thuringiensis in agricultural soils than non-agricultural soils. This could be as a result of plants serving as a form of protection to the soil from the harsh ultraviolet rays of the sun and as such the soil organisms remain intact. The bacterial isolate from diseased tomato plants was also tentatively identified as Ralstonia solanacearum. Pathogenicity assay of Ralstonia solanacearum isolate on Beske tomato variety showed wilting of varying degrees while the control showed no sign of wilting. This is in agreement with Popoola et al., (2015) who reported Beske variety as susceptible to bacterial wilt of tomato. In-vitro activity of the Bt isolates (Bt2, Bt16, Bt17, Bt32 and Bt34) against Ralstonia solanacearum revealed isolate $\mathrm{Bt} 34$ as having the highest zone of inhibition with whole cell $(20.00 \mathrm{~cm})$ and supernatant $(18.00 \mathrm{~cm})$ while isolate Bt17 had the least zone of inhibition and whole cell. Similarly, isolates Bt 16, Bt 17 and Bt 32 had the least zones of inhibition $(0.00 \mathrm{~cm})$ only. This corroborates the work of Abeer and Hend (2013), reporting that Bacillus thuringiensis was able to significantly reduce the growth of Ralstonia solanacearum under laboratory conditions with a zone of inhibition of $8.2 \mathrm{~mm}$. This result shows that native Bacillus thuringiensis may be used to control bacterial wilt of tomato under laboratory conditions.

\section{References}

Abeer, H.M. and Hend, A.H. (2013). Suppression of bacterial wilt disease of tomato plants using some bacterial strains. Life Sci.10 (3):1732-1741.

AdounignaKassogue, A., Magia,K., Traore, D., Dikko, A.H., Fane,R., Guissou, T.Faradii, F.A., Valicente,F.H., Hamadoun, A. 2015. Isolation and characterization of Bacillus thuringiensis (Ernst Berliner) strains indigenous to agricultural soils of Mal.iAfr. J. Agric. Res. 10(28), 27482755 ,

Ammons, D., Toal, G., Roman, A., RojasAvelizapa, L.I., Ventura-Suarez and Rampersad, J. (2016).Cry-like genes, in an uncommon gene configuration produce a crystal that localizes within the exosporium when expressed in an acrystalliferous strain of Bacillus thuringiensis. FEM Microbiology Letters.363(4).pii: fnw010. doi: 10.1093/femsle/fnw010.

Bergougnoux, V. (2013). The history of tomato: From domestication to biopharming, Biotechnol. adv. 32: 170-189.

Cheesbrough, M. (2006).District Laboratory Practice in Tropical Countries. 2nd Edn., Cambridge University Press, Cambridge, UK., ISBN-13: 9781139449298. 
Cleidson, V., Simone, M., Elza, F. and Artur, S. (2007).Screening methods to determine antibacterial activity of natural products.Braz. J. Microbiol.38:369-380.

Djenane, Z., Nateche , F., Amziane , M., Gomis-Cebolla , J., El-Aichar, F., Khorf, $\mathrm{H}$ and andFerr, J .(2017). Assessment of the Antimicrobial Activity and the Entomocidal Potential of Bacillus thuringiensis Isolates from Algeria .Toxins 9, 139.

Dong, Y.H., A.R. Gusti, Q. Zhang, J.L. Xu, and L.H. Zhang.(2002). Identification of quorumquenching $\mathrm{N}$-acylhomoserinelactonases from Bacillus species.Appl Environ Microbiol68:17541759.

Dun-chun, H.E., Zhan, J., Xie, L. (2016). Problems, challenges and future of plant disease management: from an ecological point of view. J IntegAgri.15(4):705-715.

El-kersh, T.A., Ahmed, A.M., Al-sheikh, Y.A. Tripet, F., Ibrahim, M.S. and Metwalli, A.A.M. (2016) Isolation and characterization of native Bacillus thuringiensis strains from Saudi Arabia with enhanced larvicidal toxicity against the mosquito vector Anopheles gambiae (s.l.). Parasites Vectors 9, 647

Elsharkawy, M.M., Nakatani, M., Nishimura, M., Arakawa, T., Shimizu, M. and Hyakumachi, M. (2015). Control of tomato bacterial wilt and rootknot diseases by Bacillus thuringiensis CR-371 and Streptomyces avermectinius NBRC14893, ActaAgriculturaeScandinavica, Section B Plant and Soil.65 (6) 575-580, DOI: 10.1080/09064710.2015.1031819.

Fawole, M.O. and Oso, B.A. (1998).Laboratory Manual of Microbiology. Spectrum Book Ltd., Ibadan, Nigeria, pp: 1-55.

Fegan, M. and Prior, P. (2005).How complex is the "Ralstoniasolanacearum species complex"? In: Allen, C., Prior, P., Hayward, A.C. (eds.). Bacterial wilt disease and the Ralstoniasolanacearum species complex.APS Press, St. Paul, MN, USA.
Holt, J.G., Krieg, N.R., Sneath, P.H.A., Stanley, J.T. and Williams, S.T.(1994). .Bergey's Manual of Determinative Bacteriology, 9th edition. Beltimore, M.D. Williams and Wilkins (eds).

Jayesh, P., Joshi, M.S., Navathe, S.andAgale, R.C. (2014).Physiological and Biochemical characters of Ralstoniasolanacearum.Int. J. Agric. Sci.1(6): 357-360.

Kempf, M.J., Chen, F., Kern, R. and Venkateswaran, K. (2005). Recurrent isolation of Hydrogen Peroxide -resistant spores of Bacillus pumilus from a spacecraft assembly facility. Astrology 5(3):391-405.

Keswani, C., Mishra, S., Sarma, B. K., Singh, S. P. and Singh, H. B. (2015). Unraveling the efficient application of secondary metabolites of various Trichoderma. Appl.Microbiol.Biotechnol. 98:533-544

Khokar, K.M. 2013).Present Status and Prospects of Tomatoes in Pakistan Agriculture Corner. DOI:

10.13140/RG.22.15944.57605.Retrieved online on $5 / 11 / 19$.

Konecka, E., Kaznowski, A., Ziemnicka, J., Ziemnicki, K. (2007). Molecular and phenotypic characterization of Bacillus thuringiensisisolated during epizootics in CydiapomonellaL. J. Invertebrate Pathology 94: 56-63.

Link, L., Sawyer, J., Venkateswaran, K. and Nicholson, W. (2004). Extreme spore UV resistance of Bacillus pumilus isolates obtained from an ultraclean spacecraft assembly facility. Microb. Ecol. 4ᄌ2): 159-63.

Marissa, M. M., Hariati, A.M. and Fadjah, M. (2016).Antimicrobial activity of Bacillus cereus and Bacillus thuringiensis on Pathogenic Vibroharveyi in Litopenaeusvannamei.LifeSci.6 (1): 10-14.

Meihiar, M., Ahmad, M., Al-Zyoud, F. and Amer, K. (2015).Environmental Distribution, Frequency and Toxicity of Bacillus thuringiensis in Syria.Annu.res.5(2): 174-183.

Mishra, R, K., Saabale, P. R., Naimuddin, K., Jagadeeswaran, R. and Mishra, $\mathrm{O}$. 
(2015).Potential Trichoderma sp. from pulses rhizosphere. Pulses newsletter, p3

Mohsina, S., Lokesh, B., Gajendra, P.S., Priyanka, S. and Preeti, S. (2013). Isolation of crystal protein from Bacillus thuringiensis. IJPAS1 (2): 44-47.

Mounyr, B., Moulay, S., and Saad, K. (2016).Methods for in vitro evaluating antimicrobial activity.A review. J. Pharm. Anal. 6: 71-79.

Neethu, K.B., Priji, P., Unni, K.N., Sajith, S., Sreedevi, S., Ramani, N., Anitha, K., Rosana, B., Girish, M.B. and Benjaminute, S. (2015). New Bacillus thuringiensis strain isolated from the gut of Malabarigoat is effective against Tetranychusmacfalanei. J. Appl. Entomol.http://doi.org/10.1111.12235

Olaniyi, J. O. Akanbi, W. B. Adejumo, T. A. and Akande, O. G.(2010). Growth, fruit yield and nutritional quality of tomato varieties. Afr.

J. Food Sci.4 (96): 398-402.

Palma, L. (2015). Protocol for the fast isolation and identification of insecticidal Bacillus thuringiensisstrains from soil.Bt Research 6 (7): 1-3.

Popoola, A. R., Ganiyu, S. A., Enikuomehin, O. A., Bodunde, J. G., Adedibu, O. B. , Durosomo, H. A. and Karunwi, O. A. (2015). Isolation and Characterization of Ralstoniasolanacearum Causing Bacterial Wilt of Tomato in Nigeria.Niger. J. Biotechnol.29: $1-10$.

Ralte, Z.O., Senthilkumar, N. and Gurusubramanian, G.(2016). Diversity and Toxicity of Bacillus thuringiensis from Shifting Cultivation (Jhum) Habitat.BiocontrolSci, Techn.21(2): 99-111.
Saadeldin, M.O. (2007). Isolation, Characterization and Nematocidal Activity of Bacillus thuringiensisIsolated from Soil and Water. A Thesis submitted to the University of Khartoum, in partial fulfillment of the requirement for M.Sc. Degree.

Shew, H.D. and Lucas, G.B. Eds. (1991).Compendum of Tobacco Diseases.APS Press, St Paul, MN: 44.

Silo-Suh, L.A(.1994).Biological activities of two fungistatic antibiotics produced by Bacillus cereus UW85.Appl. Environ. Microb.60 (6): 2023-30.

Singh, V.K., Singh, A.K. and Kumar, A. (2017). Disease management of tomato through PGPB: current trend and future perspective. 3 Biotech 7 (4): 255

Suguna, M., Xavier, R. and Sreeramanan, S. (2011). Quick isolation and characterization for the confirmation of a novel Bacillus thuringiensis strains from chicken manures. Afr. J. Microbiol. Res.5 (20): 3131-3137.

Taiwo, L.B., Ailenokhuoria, B.V. and Oyedele, A.O. (2017). Profiling Rhizosphere Microbes on the root of Maize (Zea mays) Planted in an Alfisol for Selection as Plant Growth Promoting Rhizobacteria (PGPR). Int. J. Microbiol. Res.21 (5): 1-10.

Tamura, K., Stecher, G., Peterson, D., Filipski, A. and Sudhir, K. (2013). Molecular Evolutionary Genetics Analysis Version 6.0.Mol. Biol. Evol.30 (12): 2725-2729.

Vaishampayan, P.A., Rabbow, E., Hornet, G., Venkateswaran, K.J. (2012). Survival of Bacillus pumilus spores for a prolonged period of time in real space conditions. Astrology12(5): 487-97 